\title{
Multimessenger Astrophysics
}

\author{
Francesco Salamida \\ INFN Milano Bicocca, Milano, Italy \\ currently at University of L'Aquila, L'Aquila, Italy \\ E-mail: francesco.salamidaeaquila.infn.it \\ Irene Tamborra \\ Niels Bohr International Academy, Niels Bohr Institute, Blegdamsvej 17, 2100 Copenhagen, \\ Denmark \\ E-mail: tamborra@nbi.ku.dk
}

\begin{abstract}
The past few years have witnessed exciting discoveries in particle astrophysics. Astrophysical neutrinos, with the highest energy ever observed, have been detected by the IceCube telescope, launching the high-energy neutrino astronomy era. LIGO has spotted, for the first time, gravitational waves from compact star merging. Our knowledge of the gamma-ray sky has dramatically improved thanks to the Fermi telescope. By combining information coming from such a pletora of data, we are now starting to learn about of thermal and non-thermal astrophysical sources and their properties. We here present a concise summary of the status of the art in the field as reported in the "Multimessenger astrophysics" parallel session of Neutrino Oscillation Workshop 2016.
\end{abstract}

Neutrino Oscillation Workshop

4 - 11 September, 2016

Otranto (Lecce, Italy) 


\section{Introduction}

The relation between cosmic rays (CRs), photons of different wavelengths, neutrinos and gravitational waves is at the core of investigations adopting the multimessenger approach. Figure 1 shows an overview of the currently available data sets. Because of the increasing statistics, multimessenger astrophysics will allow us to learn about the most spectacular and energetic phenomena in our universe with unprecedented precision. In fact high-energy particles carry on Earth information about the engine behind merging neutron stars, supernova bursts, active galactic nuclei, and relativistic cosmic jets.

The "Multimessenger astrophysics" parallel session of NOW 2016 presented an experimental and theoretical overview on the topic. Excellent review talks have been also delivered by speakers in the plenary sessions, such as Thomas Dent ${ }^{1}$ presenting the discovery of gravitational waves, Joanna Kiryluk describing the status of IceCube and high-energy neutrino astronomy, Felicia Krauss reviewing the gamma-ray sky as from Fermi data, Eun-Suk Seo who talked about CRs, and Nicolao Fornengo reporting about the status of multi-messenger astrophysics.

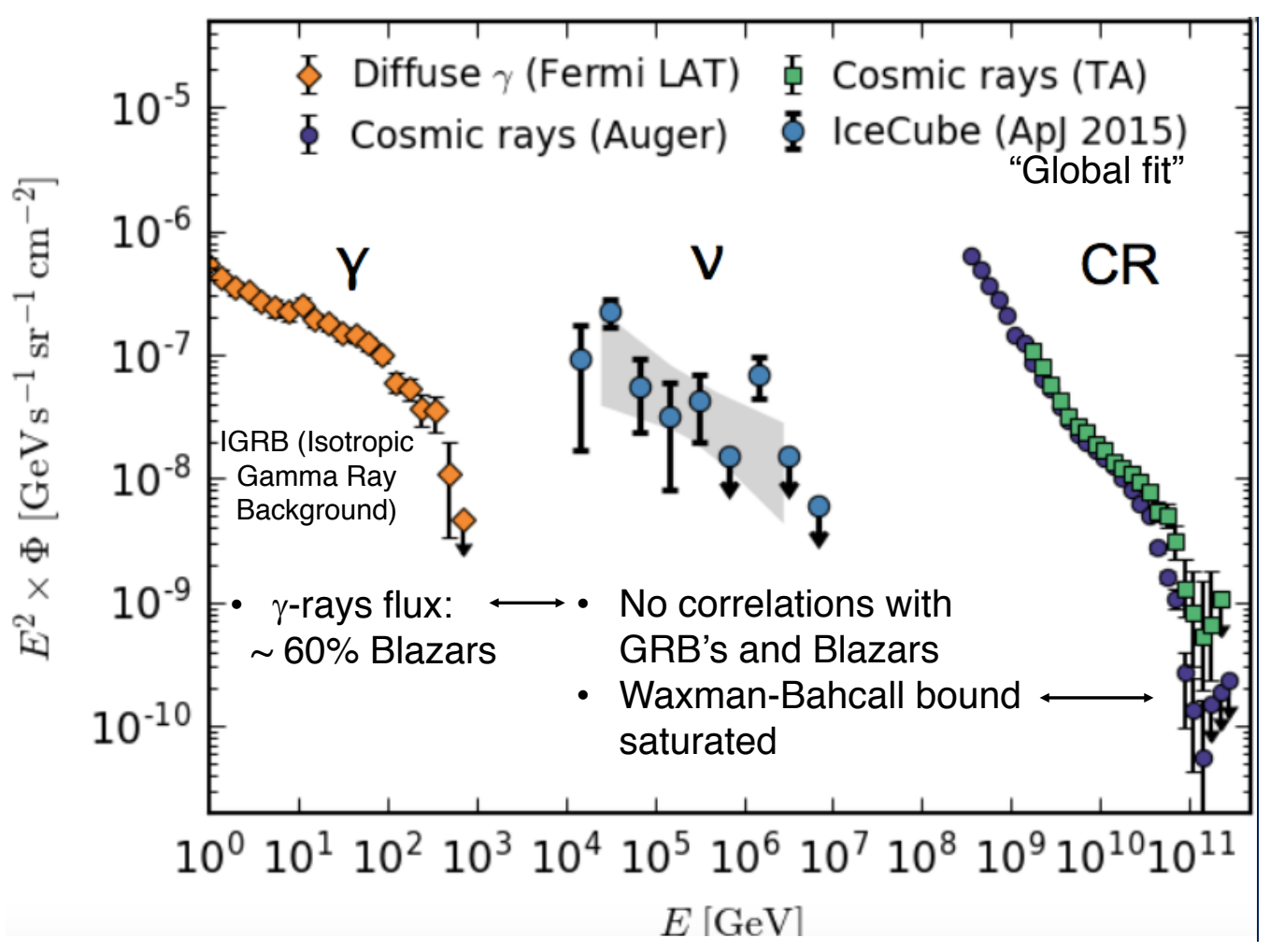

Figure 1: Multimessenger astronomy. Diffuse gamma-ray background as from Fermi data, high-energy neutrino flux detected from IceCube and cosmic ray flus as from Auger and TA as function of the particle energy. Figure taken from the talk of J. Kiryluk.

\footnotetext{
${ }^{1}$ Boldface names refer to authors of presentations delivered at NOW 2016. Their talks are available on the conference webpage http://www.ba.infn.it/ now/now2016/
} 


\section{Neutrinos from cosmic sources and their gamma-ray counterparts}

The last and only supernova neutrinos were detected from the SN 1987A. Although only about 20 neutrino events have been associated to that burst, those have proved our theory on stellar collapse and thought us about particle physics. Today several detectors around the world are in place or are under planning (or construction); they will collect high statistics from the next galactic burst. In order to extrapolate as much information as we can from the next burst, it will be crucial to combine data from liquid scintillator, water Cherenkov and liquid Argon detectors.

Among others, JUNO is a large scale scintillator detector to be built in China. As reported by Yu-Feng Li, JUNO will be almost background free and will be sensitive to all three flavors, although the main detection channel is $\bar{v}_{e}$ with about 5000 events. JUNO will allow us to reconstruct the neutrino light curve, the neutrino luminosities and average energies with high accuracy. JUNO will also provide upper bounds on the neutrino masses with a sub-eV precision and, depending on the oscillation physics, eventually on the mass hierarchy. Complementary information on the neutrino mass can also come from cosmology (see Martina Gerbino's talk).

Supernova neutrinos will be also detected with high statistics by the IceCube telescope, a neutrino detector deployed deep in the Antarctic ice. Besides low-energy astrophysical neutrinos, IceCube is already collecting an increasing number of astrophysical neutrinos with $\mathrm{TeV}-\mathrm{PeV}$ energy following the detection of Bert and Ernie, the first two PeV neutrino events (see talk by Joanna Kiryluk). Today, we count about 54 events and the statistics is constantly increasing.

The IceCube neutrinos should have astrophysical origin, although the exact sources from which they are coming from are not yet known. High-energy neutrinos are expected to be produced in cosmic accelerators trough $p p$ or $p \gamma$ interactions. Given their weakly interacting nature, neutrinos are able to escape from their sources undisturbed and therefore represent ideal probes of the physics of such extreme phenomena not otherwise investigable. The currently observed flux is close to bound estimated by Waxman and Bahcall. The flavor composition of the observed neutrinos is currently consistent at $68 \%$ confidence level with $v_{e}: v_{\mu}: v_{\tau}=1: 1: 1$ at Earth as expected for, e.g., neutrinos produced from pion decay in astrophysical sources. The flavor ratio is likely to provide information on the energy losses of pions and muons in the magnetic fields of cosmic accelerators. New physics in neutrino propagation may also modify the flavor composition.

So far an isotropic distribution of the neutrino events in the sky has been observed with no significant evidence for a spatial clustering. It is likely that the observed flux has a composite origin with the low energy part dominated by a galactic component and starbursts galaxies, and the high-energy part mostly dominated by neutrinos coming from active galactic nuclei, blazars as well as radio Faranoff-Riley galaxies, as discussed by Julia Tjus. The latter sources are indeed not very luminous but they exist in large numbers. Zurab Berezhiani also pushed forward the possibility that part of the observed flux could originate from the decay of dark matter particles. Dark matter particles with mass of few $\mathrm{PeV}$ should decay in invisible channel producing high-energy neutrinos.

The extragalactic component can be also constrained by adopting the high-energy electromagnetic emission detected by the Fermi telescope (see talk by Felicia Krauss). The sky observed by Fermi has been mostly characterized by blazars. However, up to now, only few neutrino events have been associated with a resolved blazar and with poor significance. Recent Fermi data report evidence against star-forming galaxies as dominant sources of the IceCube neutrino data. In 
fact star-forming galaxies should produce neutrinos mainly via $p p$ interactions and, assuming the observed neutrinos are mostly coming from these sources, this would allow to establish a direct relation between the diffuse gamma-ray background and the high-energy neutrino one resulting in an overshoot of the Fermi detected background. Besides blazars, choked jets and low luminosity gamma-ray bursts are plausible sources of the extragalactic neutrino flux, although scarcely constrained from gamma-ray observations. IceCube has been also searching for point sources by looking at clustering of events in a certain fixed direction. No evidence of significant correlation with known sources has been found with high significance.

As reported by Francesco Villante, the galactic contribution could make up to $12 \%$ of the detected flux. Different modelling of the galactic neutrino contribution is currently possible in the light of current CR constraints, i.e. the CR flux could be homogeneous in the Galaxy, follow the distribution of galactic cosmic ray sources such as supernova remnants and pulsars or have a spectral index that depends on the galactocentric distance. These different models may be distinguishable in a region where the galactic diffuse neutrino flux is comparable or larger than the isotropic extragalactic contribution. Although the spacial region where the galactic contribution should dominate is quite narrow, the angular distributions predicted are pretty different according to the considered scenario and in principle distinguishable by detectors with good pointing capability. IceCube and ANTARES are now approaching the sensitivity to probe some of the CR models and their respective neutrino production. KM3NeT by observing the inner galactic region with a relatively large exposure will also allow good pointing accuracy and statistics. Julia Tjus discussed the possibility that part of these neutrinos comes from supernova remnants in our galaxy.

IceCube-Gen 2, currently under design, is an extension of the current IceCube detector. IceCubeGen 2 is expected to have 10 times the IceCube volume for contained event analysis. It will aim at identifying the flavor composition and the sources of the high energy neutrinos, as pointed out by Joanna Kiryluk and Julia Tjus. A better characterization of the galactic contribution to the neutrino flux will also come from KM3NeT/ARCA. As mentioned by Giorgio Riccobene, KM3NeT/ARCA will be located at Capo Passero and it will complement the IceCube program with unprecedented sensitivity for neutrino sources. Two units of the ARCA have been already deployed and several others are under constructions. Currently the definition of the seabed network is on-going. With respect to IceCube, ARCA phase 2 will have better sensitivity for equivalent exposure and better sky coverage. ARCA is also expected to provide competitive bounds on point source searches already after 3 years.

Annarita Margiotta highlighted the role of the ANTARES neutrino telescope in the ongoing characterization of the high-energy neutrino sky. ANTARES is the largest Northern neutrino telescope and carries on searches for diffuse neutrino flux, point-like sources. ANTARES is in many ways a forerunner of the upcoming ARCA telescope and has an extensive research program, with dedicated searches also targeting the Galactic plane and the Fermi bubbles. Results on joint analysis of ANTARES and IceCube searches for point sources have also been reported.

IceCube and ANTARES are also involved in a major multi messenger effort by combining the neutrino data with electromagnetic radiation from radio, optical, x-ray,-gamma-rays, and gravitational waves observatories. Combining data from multiple experiments allows a better understanding of the related mechanisms behind the neutrino production and guarantees an increase in the detector sensitivity thanks to the suppression of uncorrelated background. 


\section{Cosmic Rays}

Some of the most interesting questions in Physics are surely related with the origin of CRs. Among the others it is very important to understand, where the transition between galactic and extra-galactic CRs is occurring, which are the accelerators and how they are transported. While it is generally accepted that galactic CRs are accelerated in shocks due to supernova explosions, it is still unclear how CRs are transported through Galaxy.

Dario Grasso has shown that high energy gamma-ray data in the inner Galactic plane as measured by e.g. Fermi and MILAGRO require to introduce new physics in CR transport models. A consistent interpretation of most updated results seems possible in terms of spatial dependent diffusion. With respect to conventional models this one predicts a significantly larger Galactic neutrino flux along the inner Galactic plane. This model could be tested usign data from IceCube, ANTARES and Km3NeT. The difference between the spectra observed in the North and Southern hemispheres is still uclear and more theoretical work, including the upgrade of propagation codes (e.g. DRAGON) is needed.

Measurements from KASCADE experiment suggest that extra-galactic CRs should vanish at energies below $10^{17} \mathrm{eV}$. Above this threshold it is important to grasp how CRs propagate through the interstellar medium.

Franco Vazza discussed the challenge to explain the origin of observed magnetic fields on $\sim$ Mpc scales. While we roughly understand how primordial energy fluctuations of the cosmic microwave background (CMB) originated cosmic structures, there is no consensus on the origin of extragalactic magnetic fields. Future experimental observations should be able to rule out alternative scenarios and constrain the distribution of magnetic fields. Moreover a possible future way to tackle this challenge is based on large magneto-hydrodynamical (MHD) simulations of cosmic magnetism by using ENZO code plus CRPropa to trace CRs propagation in the Universe.

Another approach to the propagation of CRs in the Universe has been described by Armando Di Matteo. SimProp Monte Carlo code has been used to study the propagation of ultra high energy cosmic rays in astrophysical backgrounds computing the cosmogenic neutrino fluxes expected on Earth. The study of secondary neutrinos provides a powerful tool to constrain the source models of these extremely energetic particles. It has also been showed that the detected IceCube neutrinos at $\mathrm{PeV}$ energies together with the the latest experimental results of the Pierre Auger Observatory and Telescope Array are almost at the level of excluding several hypothesis on the astrophysical sources of ultra high energy cosmic rays.

Going down from space to Earth, CRs produce particle cascades when propagating through the atmosphere. Todor Stanev reported about new features of the hadronic interaction model Sibyll 2.3 which is one of the main ingredient of cascade simulation code CORSIKA. This new implementation of Sibyll also generates charm hadrons which decay and generate very high energy atmospheric neutrinos. Simulations shows that prompt electron neutrinos can contribute to all IceCube high energy neutrino events. Moreover the predictions for charm production at LHC obtained using Sibyll 2.3 have been compared with the ones of DPMJET II code.

The Pierre Auger Observatory detects CRs at energies above $10^{17} \mathrm{eV}$ combining two different detectors (i.e hybrid mode), a set of $24+3$ fluorescence telescopes and an array of 1660 water-Cherenkov stations (SD) covering an area of about $3000 \mathrm{~km}^{2}$. Jaime Alvarez-Muñiz dis- 
cussed the most recent results of the experiment, exploiting the multi-messengers capabilities of the Observatory which can detect EeV neutrons, photons and neutrinos which have the characteristic of travelling undeflected by magnetic fields. Using the SD, neutrino induced air showers can be distinguished from air showers initiated by hadronic CRs at energies above $100 \mathrm{PeV}$. No evidence for signals from neutrons, photons or neutrinos has been found and so upper limits to the fluxes have been established, allowing to constrain UHE proton production both in galactic and extra-galactic sources. Auger is also part of the Astrophysical Multi-messenger Observatory Network (AMON), including among the others IceCube, Fermi and LIGO. In particular Auger has provided upper limits above $10^{17} \mathrm{eV}$ (complementary to those from IceCube) on Ultra High Energy neutrinos in temporal and spatial correlation with LIGO Gravitational Wave events. A major upgrade of the Pierre Auger Observatory will be completed in 2017-2018. The aim is to improve muon/electromagnetic separation in showers so enhancing the composition determination and the photon/proton discrimination power.

\section{Gravitational waves}

On September 14, 2015 at 09:50:45 UTC, the LIGO Collaboration has opened the era of the gravitational-wave astronomy by detecting the GW150914 event. In the plenary session Thomas Dent gave a very comprehensive talk on the topic.

LIGO experimental setup is made of two sites, Hanford, WA and Livingston, LA. Each one is operating a single detector, a modified Michelson interferometer measuring gravitational-wave strain as a difference in length of its orthogonal arms. Only the LIGO detectors were taking data on September 14, 2015 as the Virgo detector was being upgraded and GEO 600 was operating but not in observational mode. The basic features of GW150914 suggest it was produced by the coalescence of two black holes.

The event has been analyzed correlating the strain of the signal data with template waveforms from binary mergers with total mass 2-100 $\mathrm{M} \odot$. The templates have been fitted to post-newtonian and numerical-relativity calculations, imposing consistency between the two different detectors. The emission was found to be due to compact masses (total mass of the system $\geq 70 \mathrm{M} \odot$ ) orbiting very closely with an orbital frequency of $75 \mathrm{~Hz}$. A search for optical counterpart has been performed, but given the two experimental sites the accuracy on the position in the sky is of the order of hundreds of $\mathrm{deg}^{2}$. The extension in 2022 of the LIGO network with LIGO-India site will reduce this uncertainty.

Other two events have been reported by the LIGO collaboration, i.e GW151226 and LVT151012. No correlation has been found with neutrino events from IceCube experiment.

\section{Outlook}

Multi-messenger astrophysics is now a reality. Besides the IceCube telescope, its extension IceCube-Gen 2 is currently under design and is expected to have 10 times the IceCube volume, increasing the statistics at the highest energies and improving the identification of neutrino sources. KM3NeT/ARCA will follow ANTARES and complement the IceCube program by providing unprecedented sensitivity for neutrino sources. The sources of the observed high-energy neutrinos 
are not yet known, although blazars, starburst galaxies, low-luminosity jets, and galactic supernova remnants look plausible candidates. Those upcoming experiments will guarantee high event statistics and possibly allow to pin down the sources as well as the flavor composition of the IceCube neutrino flux, other than hopefully lead to point source detections.

The upgrade of Auger will allow to improve the knowledge on the mass composition of cosmic rays above $10^{17} \mathrm{eV}$ through a better measurement of the muon content of the showers. Hopefully ongoing studies on magnetic fields and the development of propagation codes will help to identify the sources of cosmic rays/neutrinos at the highest energies.

In the recent years the Astrophysical Multimessenger Observatory Network (AMON) project has been started. The aim of the project is to perform a real-time correlation analysis across all known high energy astronomical messengers, i.e photons, neutrinos, cosmic rays, and gravitational waves. This will enhance the combined sensitivity of collaborating observatories to astrophysical transients by searching for coincidences. By combining data coming from different cosmic messengers, we will be able to investigate the engine behind cosmic accelerators.

\section{Acknowledgements}

Support from INFN, Sezione Milano Bicocca, the Knud Højgaard Foundation, the Villum Foundation (Project No. 13164), and the Danish National Research Foundation (DNRF91) is acknowledged. The authors are grateful to the NOW 2016 organizers for their kind invitation. 